\title{
An Approach to Circumstantial Knowledge Management for Human-like Interaction
}

\author{
Alejandro Baldominos, Javier Calle and Dolores Cuadra \\ Computer Science Dept., Universidad Carlos III de Madrid, Madrid, Spain
}

\begin{abstract}
Keywords: Circumstantial Knowledge, Situation Model, Context, Context-awareness, Ubiquitous Computing, Humanlike Interaction, Natural Interaction.

Abstract: $\quad$ This paper proposes the design of a general-purpose domain-independent knowledge model formalizing and managing the circumstantial knowledge involved in the human interaction process, i.e., a Situation Model. Its design is aimed to be embodied into a human-like interaction system, thus enriching the quality of the interaction by providing context-aware features to the interaction system. The proposal differs from similar work in that it is supported by the spatio-temporal databases technology. Additionally, since the proposed model requires to be fed with real knowledge obtained from each specific interaction domain, this paper also proposes an edition tool for acquiring and managing that circumstantial knowledge. The tool also supports the simulation over the model to check the correctness and completeness of the acquired knowledge. Finally, some scenario examples are provided in order to illustrate how the Situation Model works, and to gain perspective on its future possibilities of application in different systems where context-aware services can make a difference.
\end{abstract}

\section{INTRODUCTION}

The appearance of many handheld computer devices in the last years, such as smartphones and tables, is leading to a progressive achievement of ubiquitous computing. However, the current situation still falls short of the original vision introduced by Weiser (Weiser, 1991), where computers are invisible for humans. As increasingly people are gaining access to these computer devices, the development of new interfaces is required in order to enable a simple humancomputer interaction not requiring from the user specific skills or technical capabilities; and for this reason ubiquitous computing introduces new challenges regarding the interaction with devices, which must be as simple as possible, so that the user can focus on his goals. Moreover, this interaction must attend the issue of location, meaning that it must adapt its behavior to circumstances in significant ways.

Human-like interaction (also referred as natural interaction) is a way of interaction that aims to achieve a human-computer interaction that process what comes naturally (Oviatt and Cohen, 2000) and that imitates human interactive behavior (Bernsen, 2000). This interaction style seeks to provide interfaces which attend and express human messages (uttered as if aimed at other humans) and which imitate the human interactive reasoning in order to provide access to the system services. Therefore, it turns the human-computer interaction process into a conversation between the user and the system, which should be as natural as possible (i.e. it should resemble a conversation between two humans as much as possible).

Because it resembles the interaction between humans, human-like interaction presents some attractive features that turns it into an adequate way of interaction for achieving ubiquitous computing. Particularly, it provides access to the technology to certain disabled users and to people without specific technical skills. Moreover, human-like interaction may result in an easy and comfortable interaction with the system in some scenarios, even when the user is already familiarized with the technology.

In order to achieve human-like interaction the knowledge regarding the interaction between humans must be studied and formalized so that a computer system can process it. It is generally expected that the better the knowledge is formalized, more natural the interaction would be. Due to this, research in human-like interaction involves some challenges, as it requires the study and the formalization of all the knowledge regarding the communication between humans. This includes the knowledge required for the concepts and terms regarding the interaction and the 
way they are expressed, the dialogue strategies, what is known about the interlocutors, their own beliefs, goals and emotions, the context of the interaction, etc.

This paper focuses in the circumstantial knowledge, a subset of the knowledge regarding the interaction between humans that deals with the nonlinguistical context of the interaction. In particular, the purpose of this paper is to describe the design of a general-purpose domain-independent Situation Model, a computer model storing a formalization of the circumstantial knowledge. Moreover, an intuitive edition tool is presented, which aims to handle the knowledge base of the Situation Model.

\section{THEORETICAL BACKGROUND}

Regarding an interaction process, the circumstantial knowledge deals with the context of the interaction, i.e., the set of circumstances applying in an environment. This paper focuses in this kind of knowledge, and specifically will attend the taxonomy of the context provided by Gee (Gee, 1999), which observes five different aspects of the context: (i) a semiotic aspect regarding the language used during the communication, (ii) an activity aspect concerning the transactions (or major tasks) underlying the interaction, (iii) a material aspect dealing with the physical context, such as the spatio-temporal circumstances, including people and objects taking part in the interaction, and also other environmental issues, (iv) a political aspect regarding the roles of each interlocutor and (v) a sociocultural aspect observing the influence of social and cultural conditions on the interaction.

These five aspects are not independent, and each of them influences the other. For instance, the place where the interaction is taking place may restrict the roles that each of the interlocutors can play.

Human-like interaction often represents knowledge by means of two different approaches: conversational analysis and discourse analysis (Levinson, 1983). While the first one aims at generating the interaction based on the analogy of the context with its knowledge base (e.g., with case-based reasoning), the second one focuses on the design of formal methods to produce coherent and flexible interactions. The human-like interaction system in which the proposed Situation Model will be integrated follows the discoursive approach, as it would require much less corpora than the conversational one and aims at achieving a knowledge-based (rather than samplebased) reasoning for generating the interaction.

The human-like interaction system formalizes the interaction knowledge by means of a cognitive archi-

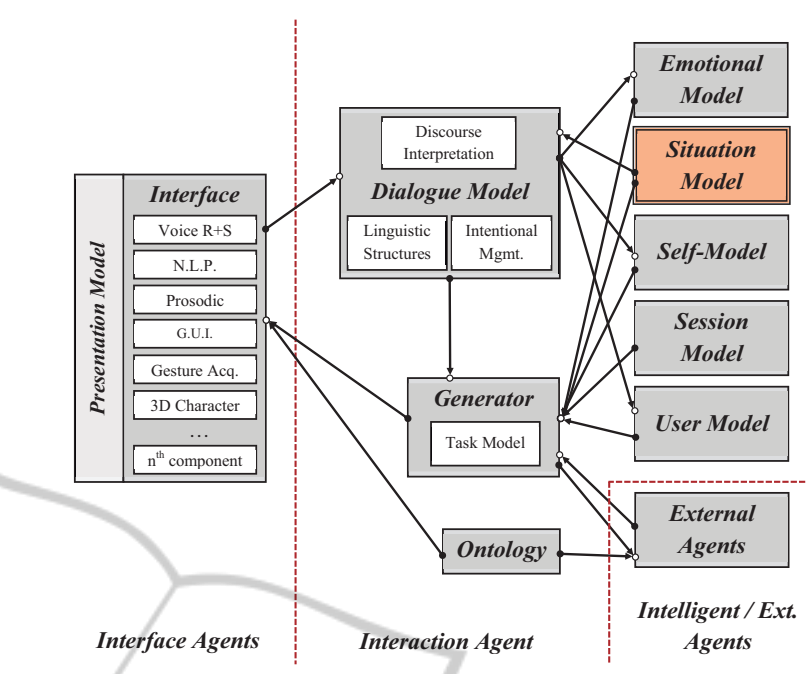

Figure 1: Cognitive architecture for human-like interaction.

tecture, i.e., a set of independent models each of them handling a certain subset of the interaction knowledge and which collaborate to produce the interaction. The next section describes the framework where the proposed Situation Model will be integrated.

\subsection{Framework}

The model-based cognitive architecture for a humanlike interaction system which will integrate the proposed Situation Model (Calle, 2004) is shown in figure 1, where each of the models deals with a subset of the knowledge regarding the interaction.

The Situation Model, dealing with the circumstantial knowledge, is highlighted in the figure. The inclusion of the Situation Model in a human-like interaction system would provide some advantages (Rivero et al., 2007), such as: (i) allowing the system to adapt itself to the current context and consequently enhancing the naturality of the produced interaction, (ii) enabling the use of circumstantial information for filtering the knowledge of other models, thus reducing the ambiguity and increasing the efficiency and efficacy of the system, (iii) providing mechanisms for situation triggering so that an action is performed when some circumstantial conditions are met, and (iv) providing new context-related functionalities such as door-to-door navigation or predictions on an object situation in the future.

Besides the inclusion of the Situation Model in the human-like interaction architecture, the proposed management tool will be incorporated to Cognos Toolkit (Calle et al., 2009; Calle et al., 2011), aimed at easing the acquisition and management of the knowledge underlying a human-like interaction system. 


\subsection{Related Systems}

This section briefly describes some of the most relevant research and commercial applications where a context model was successfully integrated within a human-like interaction system.

One of the earlier approaches is TRIPS (Ferguson and Allen, 1998) (1998), a system integrating several AI techniques in order to provide an interactive problem-solving assistant for a logistic domain. Particularly, it aims to assist the user to build plans in crisis situations which may have to meet some specific circumstantial constraints. In order to manage the circumstantial knowledge, TRIPS architecture includes a Problem-Solving Manager dealing with a database storing the problem-solving context and with specialized reasoners, whose purpose is to keep a representation of the task to be performed, the generation of a feasible plan coherent with the current circumstances and maintain the state of the proposed solution.

Secondly, introduced in 2000, Deep Map (Malaka and Zipf, 2000) is a research framework of a tourist information system which aimed to build a mobile solution offering a trip planner and a city navigator, and which would consider the user preferences and needs, and some contextual information such as weather conditions or traffic, providing an accessible interface for untrained users. Deep Map core component is the Geographical Information System (GIS), which is in charge of managing the location information, relating it to the user needs. As the user may require some historical information about a given location, GIS deals with spatial information, and a database agent extends information on specific locations by retrieving related multi-media (subject to a given moment in time, it is also able of providing historic information of past buildings or monuments in the specific location). While a third dimension stores knowledge about the visibility of objects from a given location, the fourth dimension is required to store information about the historical evolution of such objects. As the system is intended to be used in scenarios where the user may be walking or driving, Deep Map provides several interfaces, including some regarding the interaction by means of natural language for both the input and output, which turn to be unintrusive and therefore adequate in such scenarios.

In the third place, another system closed in the early 2000s is SmartKom (Wahlster, 2006), which provides symmetric multimodality to enable users accessing its functionalities. SmartKom architecture is based on models, each of them representing and reasoning about a certain type of knowledge. SmartKom is motivated for non-desktop scenarios, and for that reason the circumstantial knowledge plays a key role of the interaction process. To support the reasoning over this knowledge, SmartKom includes a context model (Porzel et al., 2006) supporting four aspects of context: dialogical (what have been said), ontological (regarding the concepts on the domain), user (knowledge about the interlocutors) and situational (the spatio-temporal context).

Finally, examples of interaction systems incorporating a context model can also be found in recent commercial applications, such as Siri, a personal assistant introduced by Apple in the iPhone 4S in 2011. This assistant enables the user asking for some information or for an action to be performed naturally. Siri takes advantage of the smartphone localization mechanisms to retrieve the user location and the Internet connection to obtain more information about the context. This enables Siri to provide context-aware services such as looking for places of interest close to the user, creating new reminders at a specified time or asking for the weather at a certain place.

While all of these models observe the material aspect of the context, they lack from a complete support for the remaining aspects of the context. Moreover, they are all designed ad-hoc for specific domains or systems, and their knowledge is introduced manually or through specific tools. While these features are not meant to be actual handicaps, they prevent the model from being reusable in other systems.

\section{PROPOSAL}

This paper proposes the design of a general-purpose domain-independent Situation Model to be embodied within the cognitive architecture for a human-like interaction system. This model will support all aspects of the context, according to the taxonomy described in section 2. Moreover, this paper describes a system for editing the knowledge stored in the Situation Model through an easy-to-use desktop application, which also serves for performing a simulation over the Situation Model in order to check the correctness of the knowledge and the model itself.

\subsection{Situation Model}

The proposed Situation Model architecture is shown in figure 2 (top). The Situation Model will be implemented over a spatio-temporal relational database (Cuadra et al., 2009), as it enables efficient storage for the location and form description of objects as well as their evolution through time. Moreover, it formalizes the circumstantial knowledge by means of 


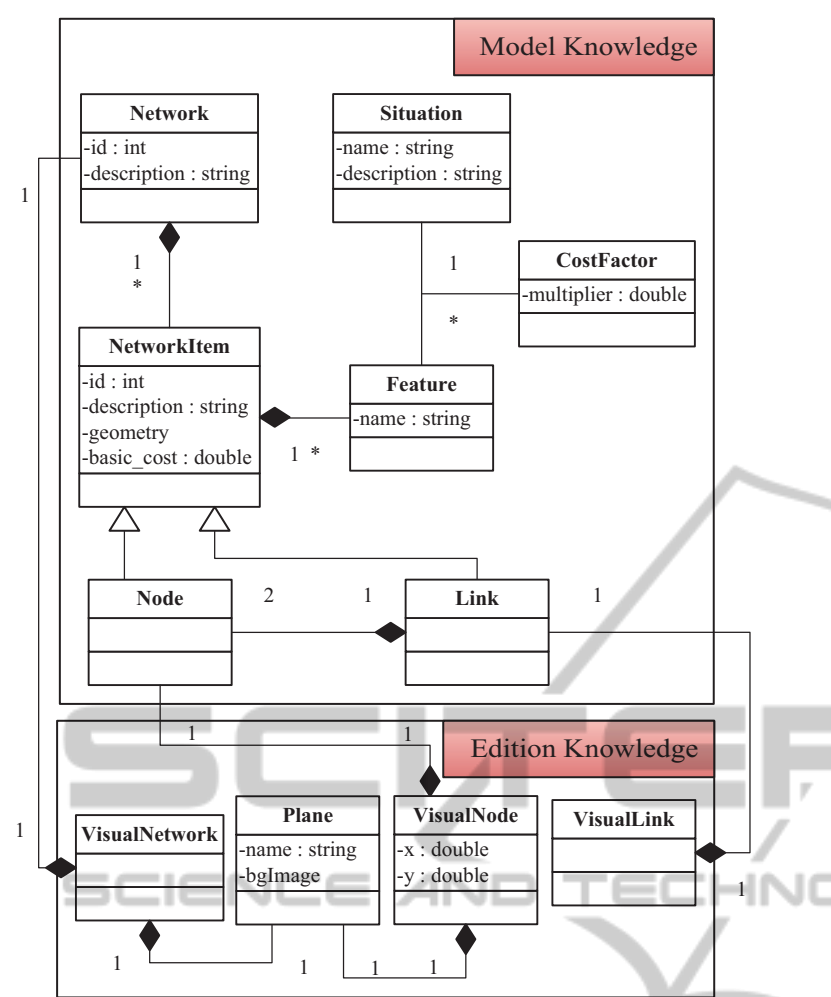

Figure 2: Architecture for the Situation Model supporting the edition.

a N-dimensional graph, a comprehensive yet powerful representation which can be easily processed by a computer system. In particular, each aspect of the context is represented by means of one or more dimensions. For instance, the spatial aspect will be represented by three dimensions, the time will add a fourth dimension and the remaining aspects will include additional dimensions. For this reason, the Situation Model is composed of networks (graphs), containing both nodes and links. The spatio-temporal database stores the geometry of each of the network items, i.e., their N-dimensional location and shape.

Furthermore, network items may have a cost, i.e., how expensive it is to traverse through them. Besides the basic cost of a network item (the one that always apply), it may also have some features modifying its cost depending on the current situations. For instance, a node placed in the stairs of a building may have a cost; however, it may implement a feature that multiplies the cost when the situation indicates that the user uses a wheelchair. Thus, the use of user-defined situations may observe any factor conditioning the context and alter the cost of going through a path depending on the existence of that factor.

Finally, figure 3 describes the way all the elements are physically interconnected by showing the physical architecture of the human-like interaction system, in-

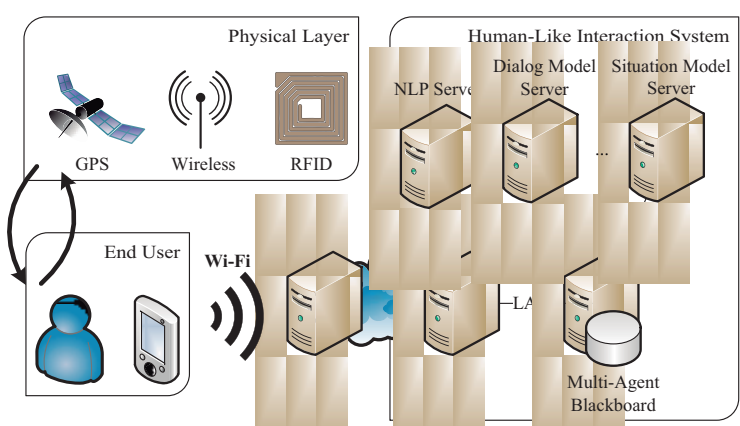

Figure 3: Physical architecture for the system.

cluding the server handling the Situation Model. In this architecture, the user carries a mobile device running the client application for the interaction system. This device is assisted by several physical layer technologies, such as GPS, RFID or Wireless; enabling the client to obtain information about the context.

The client remotely connects to the human-like interaction system, supported by a multi-agent architecture, which processes the interaction and provides the required services, including context-aware ones.

\subsection{Edition Tool}

Figure 2 shows the complete architecture of the Situation Model, including the knowledge required to edit the circumstantial knowledge. It can be seen that the Situation Model knowledge base is replicated and some additional entities supporting the edition functionalities are added. Replication provides some advantages, as the translation from the edition database to the model knowledge base is trivial. Moreover, it also enables the simulation directly over the edition model, and the proposed management tool will take advantage of this feature.

Regarding the edition model, the main entity is the visual network, serving as a wrapper for the network class of the knowledge model. A visual network is composed of planes, an entity aimed at providing a bidimensional representation from the original $\mathrm{N}$-dimensional graph, in order to show the network graphically in the edition tool. The plane may contain a background image, which would ease the placement of nodes. Finally, planes contain visual nodes, an entity serving as a wrapper for nodes and providing a virtual location for the node over the plane (this virtual location is expressed in pixels, whereas the node location may refer to actual coordinates). The visual link also serves as a wrapper for the link class, though visual links do not belong to a plane, given that a link may connect nodes in different planes.

Based on the described architecture, a management tool is proposed in order to enable the user to 


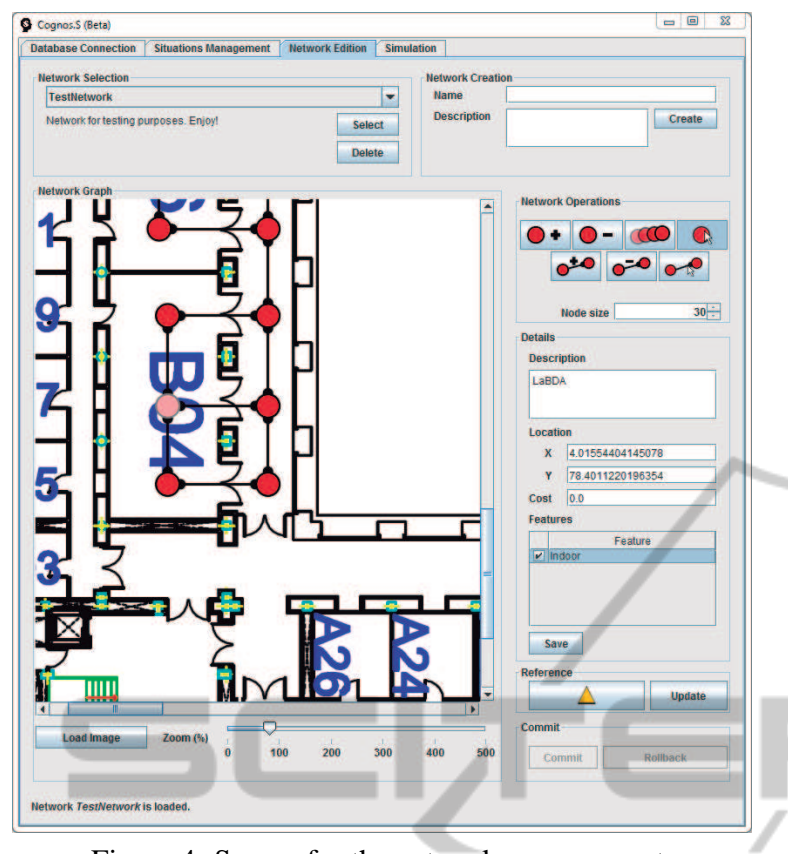

Figure 4: Screen for the network management.

manage the circumstantial knowledge. Particularly, the tool provides functionality for (i) managing situations, (ii) managing features setting cost factors to network items based on current situations, (iii) managing networks, (iv) designing of a network by placing nodes and links over a plane with a background image and (v) simulating over an existing network.

Figure 4 shows the screen which allows the management of a network, with a plane already loaded. The application allows to create, delete and move nodes and links across the plane. Moreover, a node or link can be selected in order to edit its description, location (only for nodes), basic cost or features. To ease the assignment of coordinates to the nodes, the application includes a reference feature enabling the definition of a spatial reference system by means of the definition of the coordinates of two nodes, thus inferring the location of all the other nodes once the reference system is defined. Additionally, the possibility of changing the size of the plane and of the network items independently serves for increasing the tool's accessibility. Finally, as the edition model is built over a transactional relational database, the application allows to commit or rollback the changes.

Besides the functionality for the edition of the circumstantial knowledge, the tool includes a feature to allow the execution of a simulation over the Situation Model in order to check the correctness of the acquired and formalized knowledge. The plane, which is loaded in the application, shows the network graph already edited. The user can place an agent (shown as a Pac-Man) over the plane, which can be moved through it, simulating the actual motion of a real agent. In order to perform this motion, the system emulates different technologies of the physical layer, such as RFID or GPS. The simulation over the model allows so far the execution of two different services. The description service describes the agent's current location, while the navigation service provides the user with a route between his current location and a desired target. The left panel allows the user to choose situations applying during the simulation. When situations are enabled or disabled the network items' cost change dynamically, altering the route proposed to the user.

\subsection{Preliminary Experimentation}

After the development of the edition tool, a preliminary evaluation was carried out over six subjects with different academic profiles. It is worth noting that the aim of this evaluation is to check whether this tool provides actual advantages over manual edition of the circumstantial knowledge. A complete validation of the interaction system as a whole, including the circumstantial knowledge introduced with the proposed tool, has been left for future work.

The experiment asked the subjects to feed the Situation Model with an actual network, which was printed and distributed among them. The real coordinates for each of the nodes from the network was hidden to the users, yet they were given two reference points over the network with their actual location. The experiment subjects were assigned the tasks of (i) modeling the network manually by estimating the position of each node and introducing this information into the Situation Model by means of some provided predefined SQL sentences and (ii) modeling the network through the edition tool.

For each of these tasks, the execution time was measured for each subject. Additionally, the quality of the resulting network was also measured by checking the accuracy of the modeled networks in terms of the deviation of the nodes placed by the users with respect to their original locations. The results for the objective evaluation are shown in table 1 and in figures 5(a) and 5(b). Regarding the execution time (in seconds) for both the manual edition and the edition tool, and it can be concluded that the time required by the users for modeling the network is significantly less when the edition tool is used, as the manual edition of the network is between three and four times slower. Concerning the quality of the modeled knowledge, the results show that the absolute error (the sum of the misplacements of the nodes in meters) is much lower when the network is modeled by means of the 
Table 1: Results for the evaluation.

\begin{tabular}{|c|c|c|c|c|c|c|c|}
\cline { 3 - 8 } \multicolumn{2}{c|}{} & Time & Error & Comfortable & Intuitive & Reliable & Agile \\
\hline \multirow{2}{*}{ Manual } & Avg. & 1639.5 & 10.9 & 2.33 & 2.67 & 2.83 & 2.17 \\
\cline { 2 - 8 } & St. Dev. & 283.19 & 4.62 & 1.03 & 1.63 & 1.33 & 1.6 \\
\hline \multirow{2}{*}{ Tool } & Avg. & 441.17 & 3.39 & 4.83 & 4.33 & 4 & 4.83 \\
\cline { 2 - 8 } & St. Dev. & 93.36 & 1.29 & 0.41 & 0.82 & 0.89 & 0.41 \\
\hline
\end{tabular}

edition tool, thus resulting in a higher accuracy and quality.

Regarding the subjective experiment, a questionnaire was distributed among the subjects, which were asked about the degree in which they considered each of the two different edition methods to be comfortable, intuitive, reliable and agile using a 5-point Likert scale; and also for the advantages and disadvantages of each of these methods, being able to express other considerations as well. The users' average degree of agreement with those subjectives aspects are shown in table 1 and in figures 6(a), 6(b), 6(c) and 6(d), and it can be drawn that the developed tool improves the user experience and satisfaction when performing the edition of the network.

\section{SCENARIOS}

This section describes real scenarios where the Situation Model is applied, either by itself or integrated with the human-like interaction system, which illustrate how the model can be used to assist the user or to improve the user experience with other application.

To carry out these scenarios, a smartphone application has been designed and developed, providing a navigation service which takes advantage of the Situation Model. This smartphone application allows the user to choose a target (either from a list or by speech) and to enable or disable certain situations; and it will provide the users on-screen (and also voice-synthesised) navigation instructions to reach their chosen targets. The first scenario illustrates how this mobile application would be used (further description of the tool usage is shown in http://youtu.be/ d-OsRm59z64).

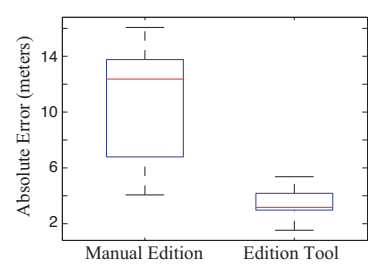

(a) Absolute error

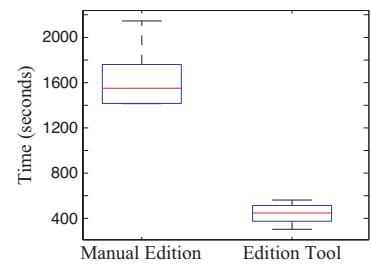

(b) Edition time.
Figure 5: Box plots for the objective evaluation.

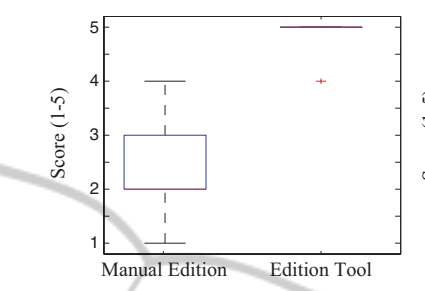

(a) Perceived comfort

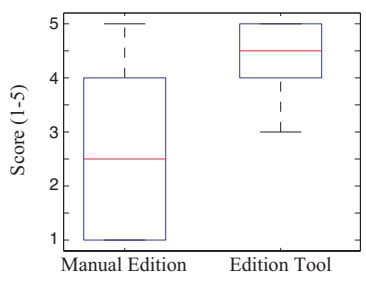

(b) erceived intuitiveness.

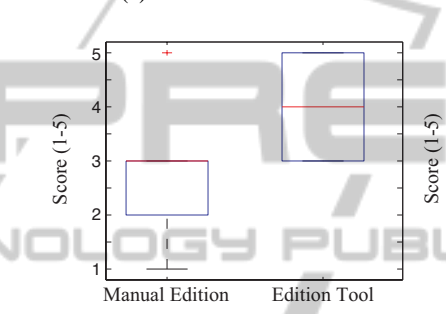

(c) Perceived reliability.

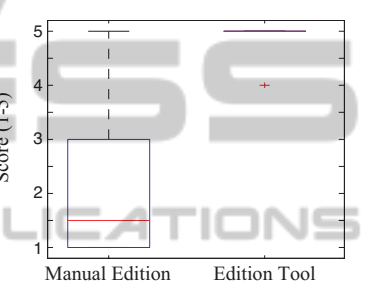

(d) Perceived agility.
Figure 6: Box plots for the subjective evaluation.

\subsection{Improving a Navigation System}

This scenario, illustrated in figure 7 shows how a pedestrian navigation system, which usually only considers the spatial aspect of the situation, can be enhanced where a broader view of the context is taken into account. For this case, we will consider that the system also observes the temporal and ambiental conditions of the material aspect of the situation. Martha (M) wants to go from the Torres Quevedo building to the Sabatini building, in the campus of Leganés of Universidad Carlos III. It is 9.00 PM and there is a heavy rain. Usually, Martha would take the shortest route, which passes by the entry of the library and enters the Sabatini park with the fountain, just before going into the building by gate A.

This route is shown in the figure as M-A. However, there are some issues for which this route is not

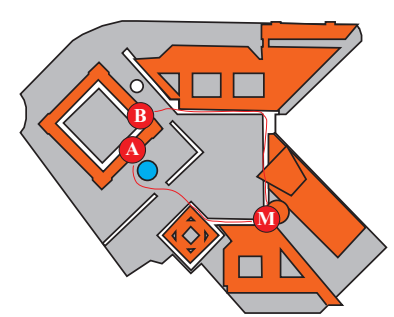

Figure 7: Map of the campus illustrating the first scenario. 


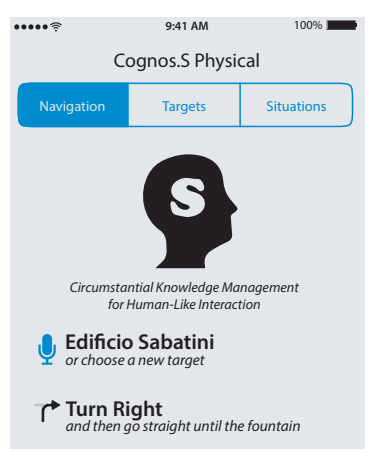

(a) Navigation screen

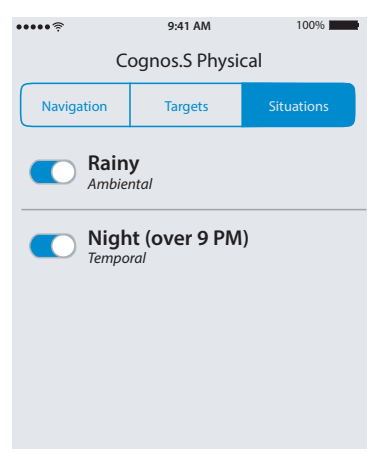

(b) Situations screen.
Figure 8: Smartphone application providing the navigation service for the Situation Model.

convenient. In the first place, entry A to Sabatini is closed after 5.00 PM, so Martha will not be able to access the building by this gate. Secondly, Martha did forget her umbrella and she really hates walking under the rain, something that wants to avoid as long as possible.

Martha, who is a very clever girl, starts her navigation system empowered by the Situation Model and asks for the best route, as shown in figure 8(a). The system is perfectly aware of the context: it knows that entry A is closed after 5.00 PM, and it also knows that the weather is really annoying for Martha (these situations are shown in figure 8(b)). Finally, the system proposes an alternative route, $\mathrm{M}-\mathrm{B}$, which goes from Martha location to entry B, which still opens at 9.00 PM. Moreover, this route is covered by the buildings, so Martha will get to her goal mostly dry. However, given that there is a small part of the route which is not covered, the system can evaluate additional options; for instance, it could start a conversation with Martha to ask whether she prefer the proposed path or waiting unless the rain is over.

\subsection{Enhancing a Natural Int. System}

This scenario shows a sample dialog between a user and a natural interaction system which is enriched by a Situation Model. While the Situation Model itself does not allow such a complex interaction, it provides an added value by incorporating context-awareness into the system, e.g., in this scenario the material and political aspects of the context are considered.

Arthur is in the building of CafreSoft Corporation, a young and promising software company. He has just finished a job interview and is really satisfied. It is 11.30 AM and it takes a few hours for him to go home, so he has decided to have his lunch in the building. He starts a dialogue with his personal assistant, Hal: ARTHUR: HAL, I'd like to eat something. What do you say?
[The system realizes it is 11.30 in the morning. It knows that ARTHUR usually have breakfast at $9.00 \mathrm{AM}$ and lunch at 1.00 PM. HAL asks ARTHUR for more information.] HAL: Nice! It's 11:30, would you have breakfast, or lunch? ARTHUR: Today I'll have something for lunch.

[The system looks for places near ARTHUR.]

HAL: There's a snack bar in this floor, you may have a sand-

wich there. Or you can go to the restaurant and eat from the menu until 3 PM.

ARTHUR: Fine, let's go there.

[HAL disambiguates the anaphora by asking ARTHUR.] HAL: To the restaurant, right? ARTHUR: Right.

HAL: Go ahead until the end of the corridor. Then go to the first floor. You can take the elevator.

ARTHUR: Nice. By the way, where can I find a bathroom?

[HAL looks for bathrooms, and finds one for staff in the floor. He looks for alternatives and assists ARTHUR.]

HAL: There's a bathroom in this floor, only for personnel.

But you can still use the restaurant restroom.

ARTHUR: Ok, I'll wait.

[ARTHUR calls the elevator.]

HAL: Remember, go to the first floor. ARTHUR: Thanks, HAL.

[ARTHUR steps out of the elevator in the first floor.] HAL: Ok, turn right and you'll find the restaurant. ARTHUR: Ok, thanks a lot!

\subsection{Assisting Disabled People}

This scenario shows how the Situation Model could take into account information about the user in order to improve its experience.

Alan is working on his University entrance exams these days. He has to do these exams in the campus of Universidad Carlos III, and he does not know the college. Unfortunately he is using a wheelchair since he was five and, while he is really used to his wheelchair, he is still having some mobility issues.

Alan has downloaded in his smartphone a mobile application developed by the University wich contains the map of the campus and includes a simple navigation feature to guide the user to a searched location. Furthermore, from the last update a simple Situation Model was implemented which takes into account, among others, some information about the user. For instance, he could select in a profile that he is a disabled person, and the system will provide alternative routes. In his case, the system will find a route that goes through ramps and elevators, rather than doing through stairs. This route will also prefer the automatic doors. Even if the route is longer and requires more time to get to the goal, the system will prefer it as long as it has less cost for Alan. 


\section{CONCLUSIONS}

This paper has proposed a design for a generalpurpose domain-independent Situation Model formalizing the circumstantial knowledge taking place in an interaction. By incorporating this knowledge to a human-like interaction system, it will be able to provide a more realistic and natural interaction, as well as specific context-aware functionalities increasingly required in the highly dynamic environments in which computer devices are used to work.

The Situation Model is implemented over a spatiotemporal relational database, which aims to provide a native support of the material aspect of the context, while the inclusion of user-defined situations also enables a partial support for the other aspects of the context. The circumstantial knowledge is formalized as a $\mathrm{N}$-dimensional graph, as it turns out to be a comprehensive yet powerful computable formalization.

Besides the Situation Model itself, a management tool is proposed to edit the circumstantial knowledge, providing means to manage networks and userdefined situations, as well as for starting a simulation over the Situation Model, which eases the validation of the edited knowledge. To support the network edition, a specific edition database was designed as a superset of the original knowledge base adding specific classes to display the network in the edition tool.

After both the Situation Model and the application were implemented, an evaluation was carried out to check whether the knowledge modeling by means of the edition tool provides actual advantages to the user. The evaluation was designed to compare the efficiency and quality of both manual modeling and the edition using the tool. In all cases, the developed application provided better results both in terms of time required by the users to complete the task and accuracy of the resulting model. Finally, a subjective survey also proved that all the users preferred the edition by means of the application regarding its reliability, comfort, agility and intuitiveness.

Future work involves designing and running an evaluation to check if the integration of the Situation Model within a human-like interaction system would actually provide the advantages described in section 2.1. To do so, the system should be evaluated before and after the Situation Model is integrated, concluding whether these advantages take place.

Moreover, a mobile application has been developed supporting real-time edition of the Situation Model. Evaluating the performance of this application and comparing it with the desktop edition tool remains as a future work.

\section{ACKNOWLEDGEMENTS}

This work has been be applied in a research project funded by the Spanish Ministry of Industry (CADOOH, TSI-020302-2011-21).

\section{REFERENCES}

Bernsen, N. O. (2000). What is Natural Interactivity? In Proceedings of the 2nd International Conference on Language Resources and Evaluation, pages 34-37.

Calle, F. J. (2004). Interacción Natural mediante Procesamiento Intencional: Modelo de Hilos en Diálogos. $\mathrm{PhD}$ thesis, Universidad Politécnica de Madrid.

Calle, F. J., Albacete, E., Sánchez, E., del Valle, D., Rivero, J., and Cuadra, D. (2009). Cognos: A Natural Interaction Knowledge Management Toolkit. In NLDB'2009, pages 303-304.

Calle, F. J., Albacete, E., Sánchez, E., Olaziregi, G., del Valle, D., Rivero, J., and Cuadra, D. (2011). Cognos: A Pragmatic Annotation Toolkit for the Acquisition of Natural Interaction Knowledge. Procesamiento del Lenguaje Natural, pages 269-276. A

Cuadra, D., Calle, F. J., Rivero, J., and del Valle, D. (2009). Applying Spatio-Temporal Databases to Interaction Agents. In DCAI'2009, pages 536-540.

Ferguson, G. and Allen, J. F. (1998). TRIPS: An Integrated Intelligent Problem-Solving Assistant. In $A A A I-98$, pages 567-572.

Gee, J. P. (1999). Introduction to Discourse Analysis. Routledge.

Levinson, S. (1983). Pragmatics. Cambridge University Press.

Malaka, R. and Zipf, A. (2000). Deep Map: Challenging IT Research in the Framework of a Tourist Information System. In Information and Communication Technologies in Tourism 2000, pages 15-27. Springer.

Oviatt, S. and Cohen, P. (2000). Multimodal Interfaces that Process what Comes Naturally. Communications of the ACM, 43:45-53.

Porzel, R., Gurevych, I., and Malaka, R. (2006). In Context: Integrating Domain- and Situation-Specific Knowledge. In SmartKom: Foundations of Multimodal Dialogue Systems, chapter 18, pages 269-284. Springer.

Rivero, J., Cuadra, D., del Valle, D., and Calle, F. J. (2007). Incorporating Circumstantial Knowledge Influence over Natural Interaction. In ICPS'2007, pages 415-420.

Wahlster, W. (2006). Dialogue Systems Go Multimodal: The SmartKom Experience. In SmartKom: Foundations of Multimodal Dialogue Systems, chapter 1, pages 3-27. Springer.

Weiser, M. (1991). The Computer for the 21st Century. Scientific American, 265:94-104. 